\title{
The latest results from the Alpha Magnetic Spectrometer on the International Space Station
}

\author{
Maura Graziani*† \\ AMS Collaboration \\ E-mail: maura.grazianiepg.infn.it
}

\begin{abstract}
The Alpha Magnetic Spectrometer (AMS-02) is a precision large-acceptance high energy particle detector. It was successfully deployed in 2011, on May 19th on the International Space Station (ISS) with the Space Shuttle STS-134 mission in order to conduct a unique long duration mission of fundamental physics research in space. AMS is able to perform an accurate measurement of the Cosmic Rays (CR) composition and energy spectra up to the TeV scale, that could reveal the presence of primordial anti-matter or give the signature of exotic sources, as for example secondaries from dark matter annihilation. An overview of the performance of the AMS-02 detector as well as the results based on data collected during the first 30 months of operations in space will be discussed.
\end{abstract}

53rd International Winter Meeting on Nuclear Physics,

26-30 January 2015

Bormio, Italy

*Speaker.

${ }^{\dagger}$ INFN and University of Perugia, I-06100 Perugia, Italy 


\section{The AMS-02 Detector}

The Alpha Magnetic Spectrometer (AMS) is a large acceptance cosmic ray detector which has been installed during the STS-134 NASA Endeavour Space Shuttle mission on the International Space Station (ISS) in May 2011, where it will collect cosmic rays until the end of the ISS operation, currently set to 2024. Thanks to the long exposure time combined with a large detector acceptance $\left(0.5 \mathrm{~m}^{2} \mathrm{sr}\right)$, AMS is able to study the primary CR fluxes in the energy range $1 \mathrm{GeV}$ $1 \mathrm{TeV}$ with unprecedented precision and sensitivity.

The main goals of the experiment are the direct search of anti-nuclei and indirect search of dark matter particles through their annihilation into light particles such as $\bar{p}, e^{ \pm}$, or $\gamma$-rays [1], gamma ray astrophysics and exotic physics (strangelets). Furthermore, the AMS-02 data are expected to significantly improve our understanding of the $\mathrm{CR}$ acceleration and propagation processes in the Galaxy $[18,19]$. The measurements of low energy CR fluxes during an entire 11 years solar cycle will also help in the understanding of solar physic and in the propagation of CRs in the Heliosphere.
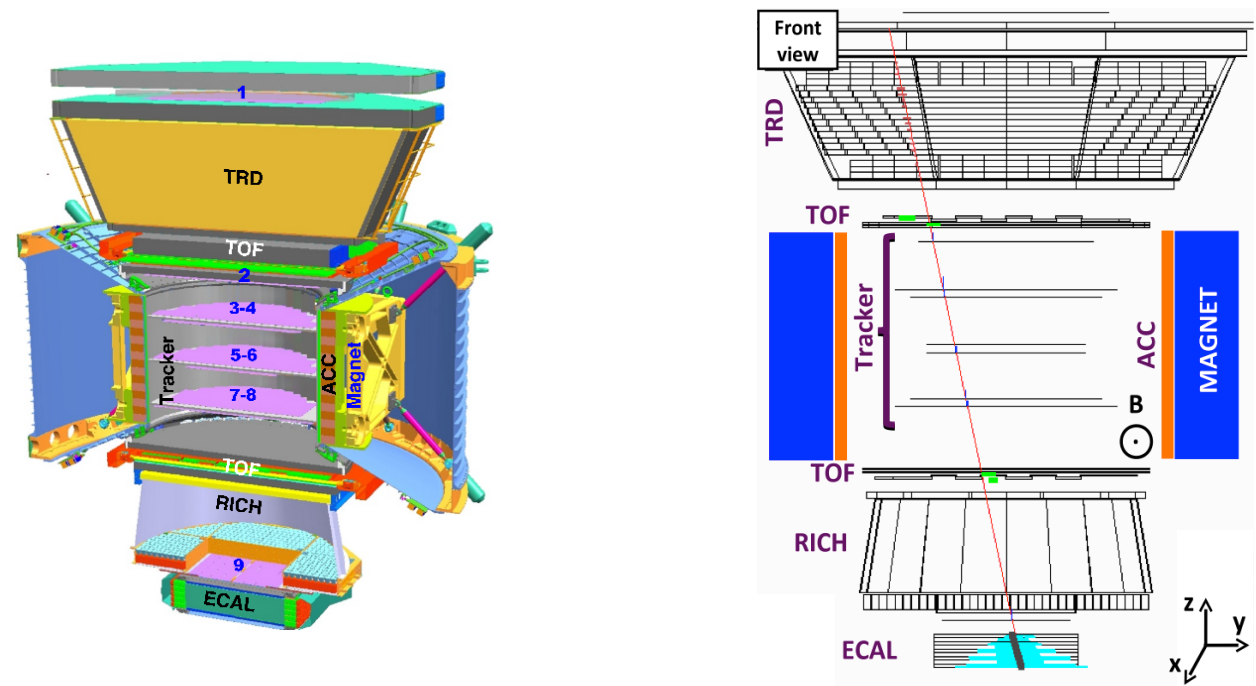

Figure 1: On the left: schematic view of the AMS-02 spectrometer. On the right: a $660 \mathrm{GeV}$ electron measured by the AMS detector on the ISS in the bending $(y-z)$ plane. Tracker planes measure the particle charge and momentum. The TRD identifies the particle as an electron. The TOF measures the charge and ensures that the particle is downward-going. The RICH independently measures the charge and velocity. The ECAL, independently identifies the particle as an electron and measures its energy.

To reach its scientific goals, the instrument - which schematic layout is reported in Figure 1 has been conceived with a large redundancy in the measurement of particle properties with complementary techniques by different sub detectors. AMS- 02 has a volume of $5 \times 4 \times 3 \mathrm{~m}^{3}$, weights 7.5 tons and was built taking advantages from the experience of the high energy particle physics experiments. 
The core of the instrument is a spectrometer, composed of a permanent magnet, which produces a magnetic field with an intensity of $0.14 \mathrm{~T}$, and of 9 layers of double-sided micro-strip silicon sensors. The task of the spectrometer is the reconstruction of the trajectory and the measurement of the rigidity $(R=P / e Z$, momentum/charge ratio).

Above and below the spectrometer two planes of time of flight counters (ToF) provide the main trigger of AMS-02 and distinguish between up-going and down-going particles. This information combined with the trajectory curvature given by the spectrometer, is used to reconstruct the sign of the charge. A Transition Radiation Detector (TRD) is located at the top of the instrument. The detector is completed with a Ring Imaging Cherenkov detector (RICH) and an electromagnetic calorimeter (ECAL).The central part of AMS-02 is surrounded by an anti-coincidence system (ACC).

The AMS-02 detector is described in details in [1].

In the following will be presented the latest results of AMS of electrons and positrons in the cosmic rays and will be briefly discussed the measurement techniques which have been used.

\subsection{The importance of the electronic component in Cosmic Rays}

The electronic component of CR carries important physics information. Due to their low mass, electrons $\left(\mathrm{e}^{-}\right)$and positrons $\left(\mathrm{e}^{+}\right)$are subject to important energy losses in the interaction with the Inter Stellar Medium, during their trajectory between the sources and Earth. For this reason they carry information about the origin and the propagation of CR complementary to the hadronic component. Due to their strong energy losses, electrons and positrons at high energies are unique probes to study the CR source property in the galactic neighborhood [12].

An excess of electrons in the range $300-700 \mathrm{GeV}$ with respect to the expected spectrum from conventional diffuse electron sources has been reported by ATIC [13] and PPB-BETS[14]. The following measurements of FERMI [10,11] observed a spectral flattening of the $\mathrm{e}^{-}+\mathrm{e}^{+}$spectrum between 70-200 GeV and a small excess at higher energies with respect ATIC and PPB-BETS. At higher energies, a rapid steepening of the spectrum is observed by HESS $[15,16]$. The PAMELA measurements of the positron fraction [9] and the $\mathrm{e}^{-}$spectrum[17] have pointed to the need of a fresh source of electrons and positrons contributing to the observed features in the high energy part of the $\mathrm{e}^{-}, \mathrm{e}^{+}$spectrum.

The measurement of the cosmic electrons $\left(\mathrm{e}^{-}\right)$and positrons $\left(\mathrm{e}^{+}\right)$is challenging: charged cosmic rays between $1 \mathrm{GeV}-1 \mathrm{TeV}$ observed at Earth, are made substantially of protons ( $\sim 90 \%)$, Helium $(\sim 8 \%)$ and heavy nuclei $(\sim 1 \%) . \mathrm{e}^{-}$and $\mathrm{e}^{+}$constitute respectively $(\sim 1 \%$ and $\sim 0.1 \%$ of the total CR flux. The main challenge in the measurement of the electronic component is the natural high background/signal ratio. The ratio to the main CR component, i.e. protons (p), amounts to $\mathrm{e}^{-} \mathrm{p} \sim 10^{-3}-10^{-2}$ and $\mathrm{e}^{+} / \mathrm{p} \sim 10^{-4}-10^{-3}$ depending on the energy.

In order to obtained the needed high e/p rejection power, AMS-02 uses mainly three sub detectors: The Radiation Transition Detector (TRD), the electromagnetic calorimeter (ECAL) and the Tracker (TRK). 


\section{Rigidity measurement and Charge Confusion}

The Silicon Tracker is made of 2264 double-sided micro-strip silicon sensors distributed over 9 layers [5,6] with an active area of $\sim 6.4 \mathrm{~m}^{2}$. As show in Figure 2, 3 of the 9 layers are located outside the magnetic field of the permanent magnet: one is located on top of the TRD, another one above the magnet, and the last one between the RICH and the ECAL. The others 6 layers constitute the inner Tracker which is contained in the permanent magnet field volume.

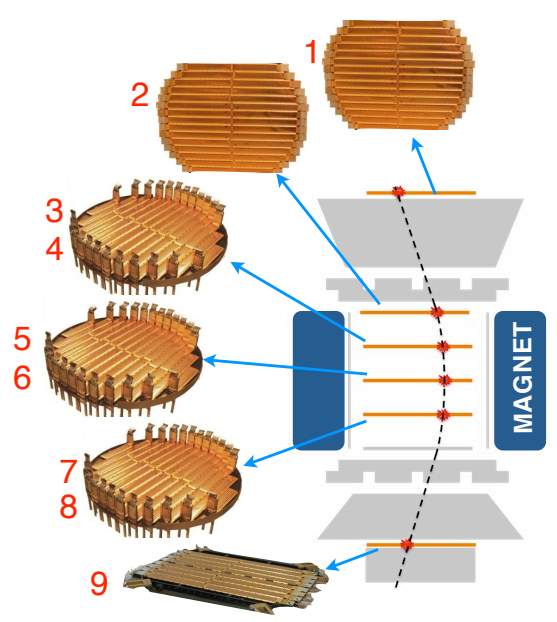

Figure 2: Disposition of the 9 Silicon Tracker layers: 3 are located outside the magnetic field. The others 6 layers constitute the inner Tracker which is contained in the permanent magnet field volume.

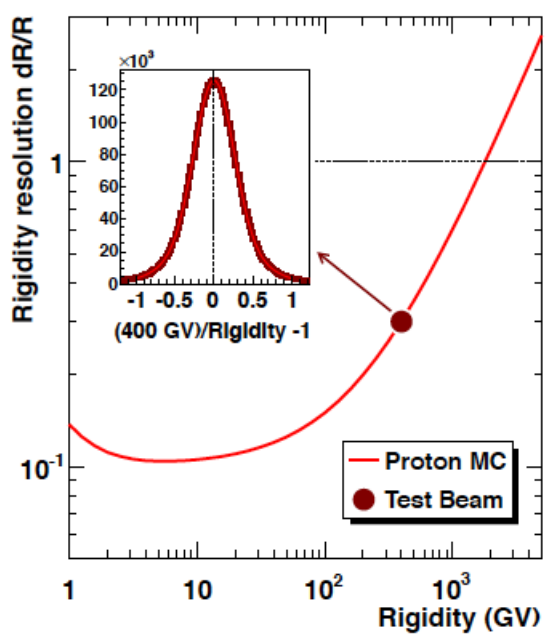

Figure 3: Rigidity resolution as estimated by Monte Carlo protons. The MDR is $\sim 2 \mathrm{TeV}$. The result from $450 \mathrm{GeV}$ Test Beam protons is superimposed (full red circle) validating the simulation.

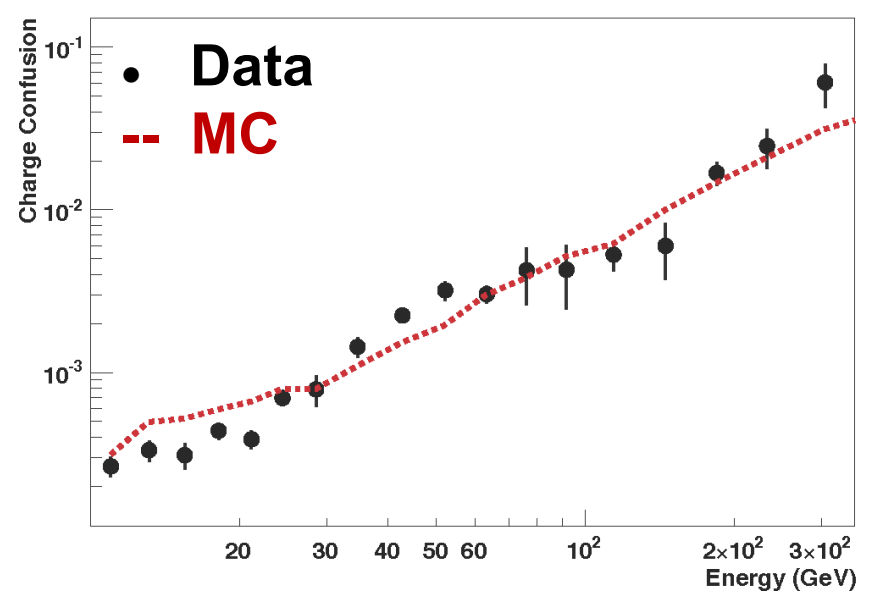

Figure 4: Charge confusion for electrons and positrons. The expectation from Monte Carlo (red dashed line) and the measurement, directly from ISS data (full black circles), are shown. 
The tracker is able to detect the crossing points of particles with high accuracy ( $\sim 10 \mu \mathrm{m}$ in the bending direction and $\sim 30 \mu \mathrm{m}$ along the non-bending one). From the crossing points it is possible to reconstruct the trajectory of the particle and the curvature of the trajectory provides the measurement of the particle Rigidity. Continuous monitoring of the tracker stability is performed in flight with a internal laser alignment system and by cosmic rays allowing to maintain the tracker alignment uncertainties at the few microns level to reach in space the same performances verified on ground.

It has a Maximum Detectable Rigidity, MDR, of $\sim 2 \mathrm{TeV}$ for single charged particles as presented in Figure 3 reporting the rigidity resolution for proton particles estimated with a Monte Carlo simulation of the full detector and validated with beam test (red point in the figure) before launch. The accuracy of the rigidity measurement crucial to measure the momentum of all the non-electromagnetic particles (see Sec.3) as protons and nuclei, up to the TeV.

Combining the information about the direction of the particle given by the ToF (down-going or up-going particle) with the sign of the rigidity is possible to reconstruct the sign of the charge. Given the orders of magnitude in the relative abundances between matter and anti-matter in the various species (protons/anti-protons $\sim 10^{4}$, electrons/positrons $\sim 10$, Helium/anti-Helium $>10^{9}$ ), the misidentification of particles charge sign, Charge Confusion (CC), may have a relevant effect on the accuracy of the measurements. The $\mathrm{CC}$ can be generated by to two main effects: the spillover and the wrong hit pickup. The spillover is the dominant source of $\mathrm{CC}$ for high momentum particles: the curvature radius of the track inside the magnetic field increases with increasing particle momentum and, due to the finite measurement resolution of the trajectory, it becomes more probable to reconstruct the trajectory with a flipped curvature sign. At lower energies, the largest source of CC is related to spurious hits in the tracker - related to noise or interactions in the detector material - which are erroneously associated to the particle track in the pattern recognition, inducing a wrong reconstruction of its trajectory hence of its rigidity value and charge sign. This effect can be mitigated searching for the effects of the interactions in the other sub detectors, once their topology in the instrument is known they can be either rejected or statistically subtracted based on their characteristic distributions.

In Figure 4 the $\mathrm{CC}$ for electrons and positrons, as a function of energy is shown. The expectation from MC is compared with the CC measured directly from data: to measure the amount of $\mathrm{CC}$, a template fit approach has been adopted by looking at the activity in the detector and at the energy/rigidity ratio.

\section{The measurement of the energy}

High accuracy measurement of the particle energy is achieved for electromagnetic components of the cosmic radiation by means of the ECAL.

The ECAL is a sampling electromagnetic calorimeter with an active area of $64.8 \times 64.8 \mathrm{~cm}^{2}$ and 17 radiation lengths thickness [8]. It is made by 9 superlayers, each layer is composed by lead interleaved with scintillating fibers that run in one direction only. The superimposition of superlayers with fibers running parallel to the $\mathrm{X}$ and $\mathrm{Y}$ axes (five and four superlayers, respectively) allows the ECAL to reconstruct the 3D development of the shower. 
The energy resolution of ECAL is evaluated from beam tests of the complete AMS-02 detector and is parametrized as a function of energy $\sigma(E) / E=\sqrt{(0.104)^{2} / E+(0.014)^{2}}$. The resolution as a function of energy is shown in Figure 5 superimposed with the results coming from Test Beam electrons.
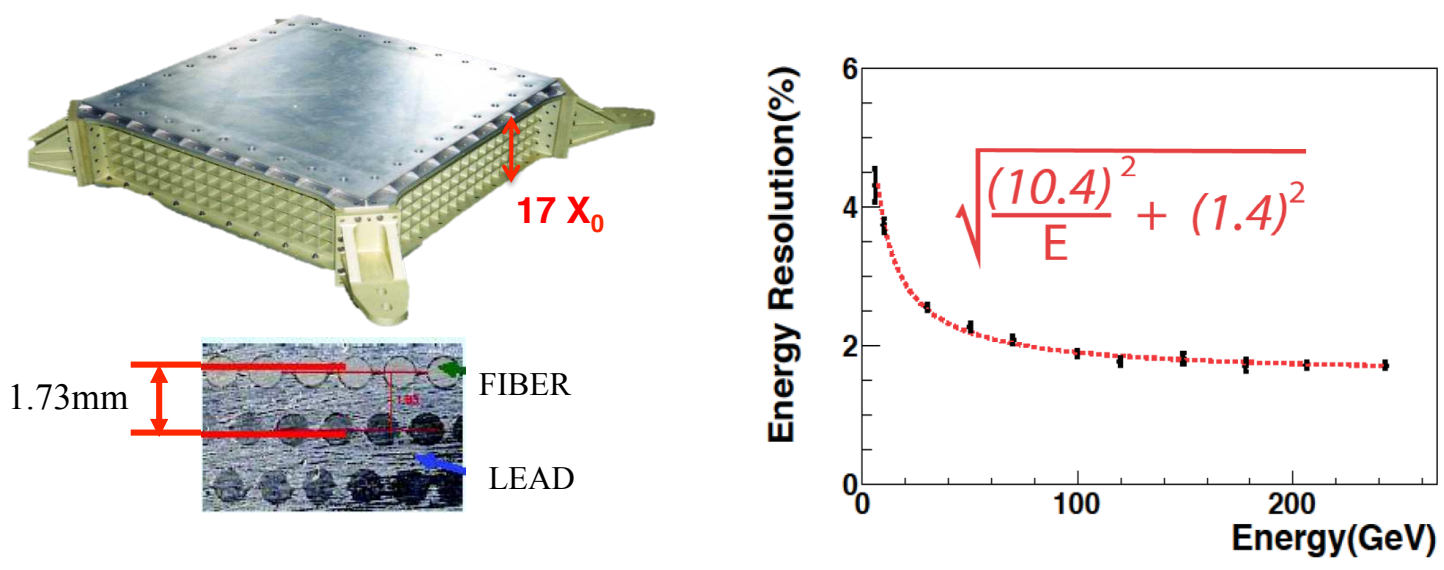

Figure 5: On the left, The AMS-02 Electromagnetic CALorimeter. It is made of lead interleaved with scintillating fibers and its deep corresponds to $17 \mathrm{X}_{0}$. On the right, The ECAL resolution as a function of energy (red dashed line) superimposed with the results from Test Beam electrons (black points).

\section{Electron-proton separation}

The two key detectors for the lepton-hadron separation are the TRD and the ECAL. These detectors allow the rejection of the overwhelming background coming, mainly, by CR protons, and are used to perform the measurement of the leptonic components.

\section{1 e/p separation with the Transition Radiation Detector (TRD)}

The key point in the working principle of the TRD is the efficient detection of transition radiation (TR) - a soft X-ray emission - with the smallest amount of material in the particle path. The TRD is constituted by 328 modules arranged in 20 layers [7]. Each module contains $20 \mathrm{~mm}$ of radiator made of polypropylene/polyethylene fiber fleece and 16 tube straws filled with a 90:10 $\mathrm{Xe}: \mathrm{CO} 2$ mixture. In the radiator material the refraction index changes several times. This increases the probability for the crossing particle to emit transition radiation $\mathrm{X}$-rays. The emission probability is proportional to the Lorentz boost factor $\gamma$, therefore electrons produce more transition radiation than protons with the same energy.

The ADC spectrum released by electrons and protons inside one TRD tube is shown in Figure 6 (on the left). For protons, only the ionization spectrum is visible. For electrons, instead, the TR spectrum corresponding to higher energy release is superimposed on top of the ionization spectrum. 

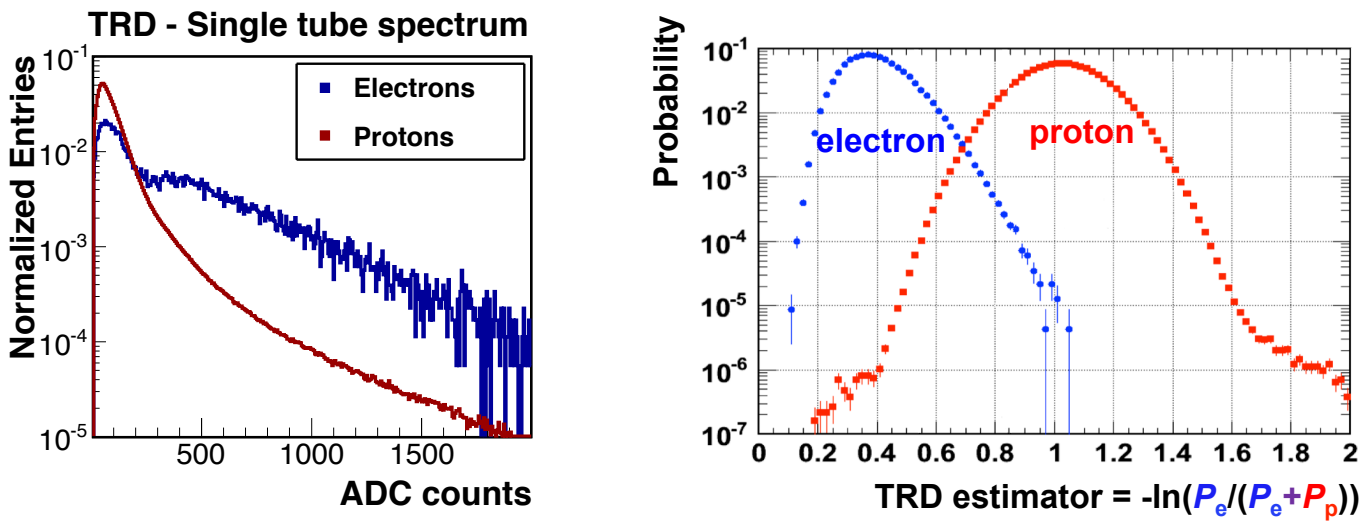

Figure 6: On the left, TRD single tube ADC spectra for $25 \mathrm{GeV}$ electrons (blue) and protons (red) selected from ISS data. Protons only lose ionization energy in the tubes. Electrons, instead, can produce TR X-rays with consequent higher energy release, visible in the right tail of the distribution. On the right, a TRD estimator, defined by likelihood electrons $\left(P_{e}\right)$ and protons $\left(P_{p}\right)$ ratio, used to separate electrons from protons.

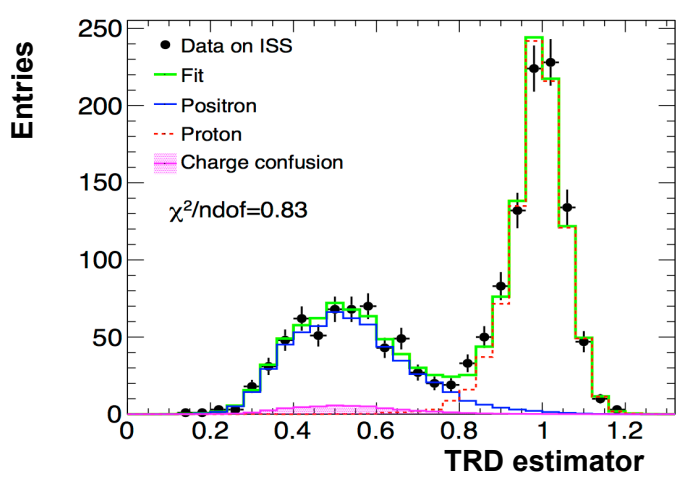

Figure 7: Fitting method applied on TRD estimator in order to statistically estimating the electrons(positrons) component in the sample.

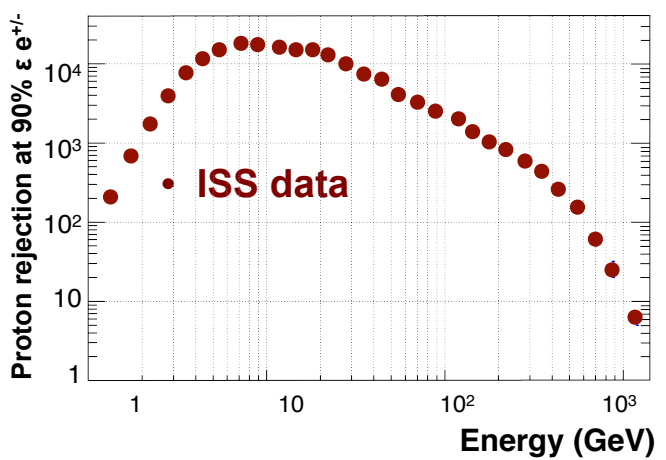

Figure 8: The proton rejection, as a function of energy, with a $90 \%$ efficiency cut on the TRD estimator.

The observed signals in all TRD layers associated to the reconstructed particle are combined in a log-likelihood estimator for the electron $\left(\mathrm{P}_{e}\right)$ and proton $\left(\mathrm{P}_{p}\right)$ hypothesis. The two likelihoods can be used (for example by means of a likelihood ratio approach, as shown in Figure 6, on the right) to separate electrons from protons using fitting method in order to statistically estimating the electrons(positrons) component in the sample. In this case, the reference spectra of the TRD estimator has to be retrieved in each energy bin directly from data: in order to obtained the electrons TRD template, it is possible to use the ECAL BDT (see section 4.2) in order to remove the proton background, instead for the definition of proton template is more simple since $p$ are the dominant cosmic ray species. The TRD classifier reference spectra are used to statistically separate the signal $e^{ \pm}$contribution from the $p$ background as show in Figure 7. 
The achieved proton rejection, as a function of the energy, is shown in Figure 8.

\section{2 e/p rejection with the Electromagnetic Calorimeter (ECAL)}

In order to cleanly identify electrons and positrons, a statistical estimator (Boosted Decision Tree algorithm [8]), has been deployed by means of the different characteristics between electromagnetic and hadronic showers. The estimator combines 19 variables from the imaging reconstruction of the electromagnetic shower. The distribution of the BDT estimator for electrons and protons is shown in Figure 9.

Moreover, the ratio between the energy measured by the ECAL $(\mathrm{E})$ and the rigidity measured by the Tracker can be used to reject the proton background $(\mathrm{E} / \mathrm{R} \sim 1$ for electrons and positrons , $\mathrm{E} / \mathrm{R} \sim 0$ for protons).

The achieved proton rejection with a 90\% efficiency cut on the BDT, combined with a cut on the matching between energy in ECAL and momentum in the spectrometer, $\mathrm{E} / \mathrm{p}<0.75$, is shown in Figure 10 (right).

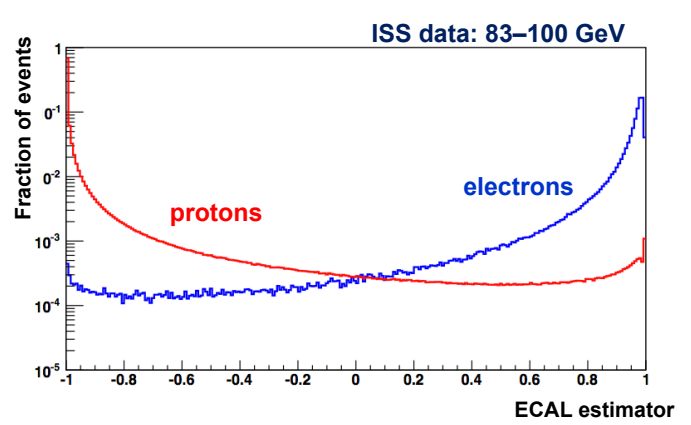

Figure 9: ECAL Boosted Decision Tree estimator for protons (red) and electrons (blue) in the energy range $80-100 \mathrm{GeV}$.

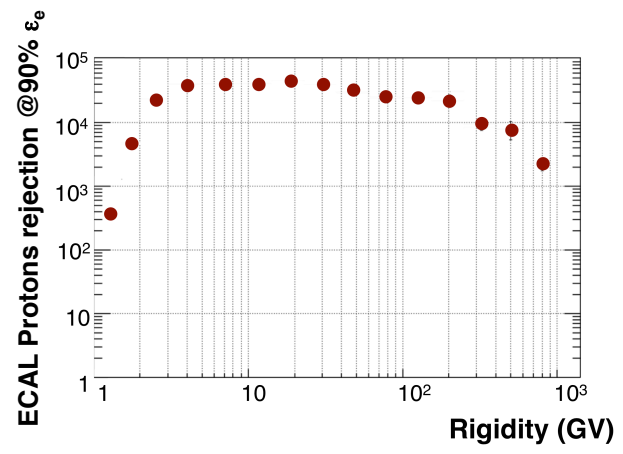

Figure 10: The proton rejection, as a function of energy, with a $90 \%$ efficiency cut on the BDT combined with a E/p $0.75<$ cut.

\section{Charge Measurements}

Information on the $\mathrm{CR}$ nuclear charge $Z$ are obtained by the multiple measurements of energy loss in the several detectors of the spectrometer and by the amount of Cherenkov light detected by the RICH. Inside the TRD, the ionization energy loss in the $\mathrm{Xe} / \mathrm{CO}_{2}$ gas mixture of each of the 20 layers is proportional to $\mathrm{Z}^{2}$ and can be used to measure the nuclear charge. The same technique is used also in the 4 layers of plastic scintillators of the TOF and in the nine tracker layers. In the RICH, instead, the value of the charge is determined by the number of photons counted in the Cherenkov ring. Even in the ECAL, the energy loss for MIPs can be used to measure the Z.

The adopted analysis strategy is to identify the CR nuclear charge using the 7 Inner-Tracker layers (from L2 to L8) and the 4 TOF planes, while complementary information from the other subdetectors are used in a second stage. The Inner Tracker+TOF combination represents the basic 

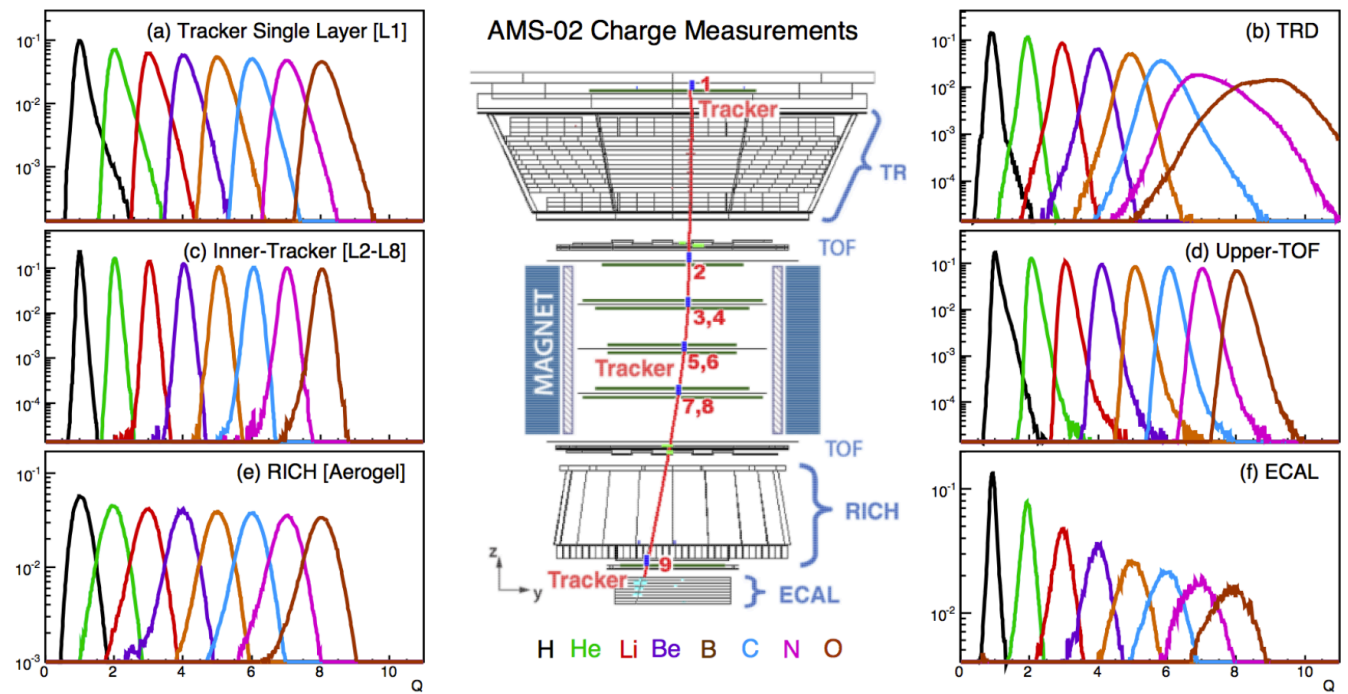

Figure 11: Schematic Y-Z view of the AMS-02 detector, illustrating the path of a typical CR event. The panels show the charge response of single sub-detector units to light CR elements $(Z=1$ to $Z=8$ ): (a-c) Tracker, (b) TRD, (d) TOF, (e) RICH, and (f) ECAL.

core of the AMS-02 spectrometer and ensures charge separation capabilities over a wide dynamical range $(Z=1-26)$. In the Silicon tracker, thanks to its double sided nine layers and the high dynamic range of the front end electronics, the energy loss can be measured with high accuracy. The overall Silicon Tracker resolution for the charge is $\sim 0.1$ c.u., and this is crucial to measure the relative abundances of all the nuclei up the Iron $(\mathrm{Fe})$. Each single layer, if used as a standalone detector, has however a very good resolution, $\sim 0.3$ c.u.. Such a good resolution with a single measurement is very important especially for the first tracker layer. Being at the top of the instrument, it allows the detection of the events entering the detector with a certain charge and, due to the fragmentation process, arriving in the lower sub-detectors, as the inner tracker, with a different (lower) one. This capability of tagging the fragmentation is very important for an accurate measurement of all the nuclei, especially the less abundant species where fragmentation in the detector of other species could spoil their flux measurement accuracy.

The single charge estimators are shown in Figure 11 where each plot is referred to a specific sub-detector where the various $C R$ elements $(Z=1-8)$ have been identified using information from other sub-detectors.

\section{Thermal environment and TTCS system}

The space thermal environment of AMS is different from the ground-based accelerator experiment or satellite-based experiment. On board of the ISS, AMS orbits around the Earth with a period of $\sim 93$ minutes and is subjected to extreme thermal variations due to day/night effect during its orbits and seasonal Sun exposure variation or due to changes of the attitude of the ISS, as in the case of berthing/unberthing of visiting vehicles [20]. Since each AMS sub-detector has its own warning, operational and no-operational temperature range, AMS requires constant alertness in order to avoid damage to its electronics. For this purpose, several sensors are placed on each 
sub-detector and the temperatures of AMS are constantly under monitoring.

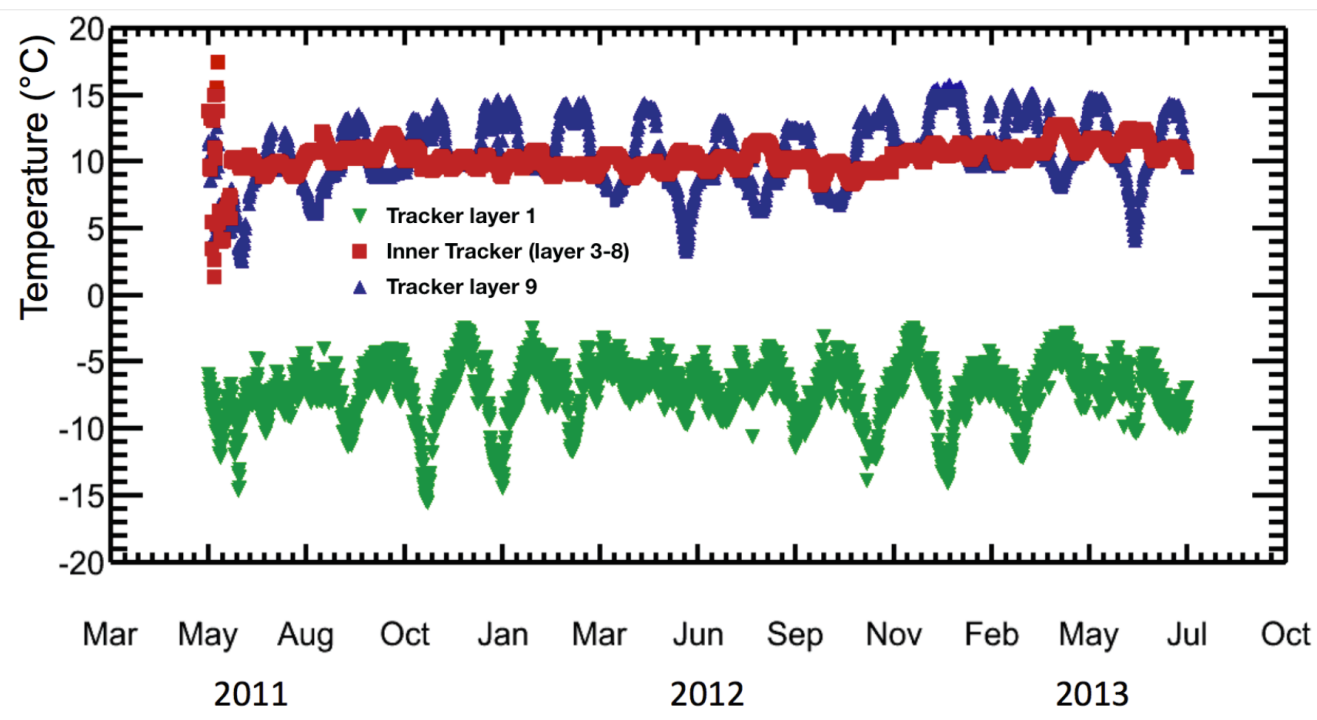

Figure 12: Time evolution of the temperature measured in different sensors thermally connected to the tracker front-end electronic of the Inner Tracker, layer 1 and layer 9.

In particular, the Tracker electronics, that produces almost the whole quantity of the AMS electronics heat, has its own temperature control system: the TTCS (Tracker Thermal Control System). The AMS-02 TTCS is a mechanically pumped two-phase $\mathrm{CO}_{2}$ cooling loop. It is able to remove $140 \mathrm{~W}$ of heat by means of two dedicated radiators facing outer space located on the top of AMS-02. TTCS provides also stability with respect to the environmental temperature described above.

In Figure 12 is shown the average temperature of the Tracker layer 1, Inner Tracker and tracker layer 9 during the first two years of operation. Since the inner tracker and layer 9 are cooled by the TTCS system, their temperature has a small variation with respect to the orbital thermal environment changes. The wide temperature variations that can be observed at the beginning of AMS-02 operations in 2011 were due to the commissioning of the TTCS system on orbit. Since the Tracker layer 1 is facing outer space, it has no need of a cooling system but a system of heaters has been installed on this layer to warm it up in the case of too low temperatures.

\section{The Data sample}

The detector is orbiting around the Earth at an altitude of $\sim 400 \mathrm{~km}$ from ground and performs a complete orbit in $\sim 93$ minutes. Figure 13 shows the acquisition rate as a function of the geographical coordinates (left) and the corresponding live time (right).

Up to the January of 2015, in about 44 months in orbit, AMS had collected $\sim 60$ billion cosmic rays events. In this proceeding, are reported the latest published results of AMS, based on the data collected during the first 30 months of operations on the International Space Station (ISS), from 19 May 2011 to 26 November 2013. This corresponds to the exposure time shown in Figure14. 

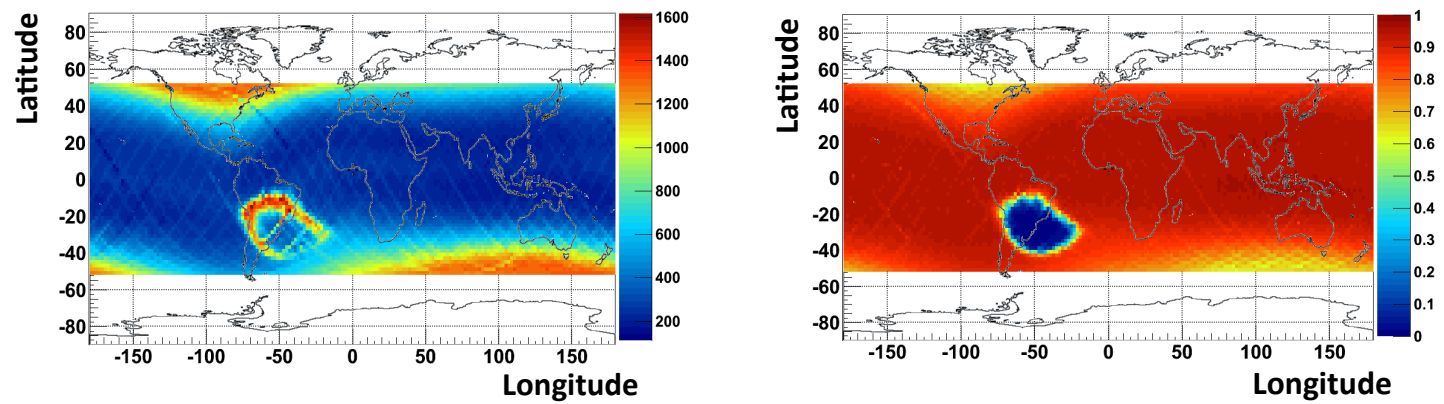

Figure 13: (Left) Acquisition rate as a function of the geographical coordinates. The average acquisition rate $\sim 500 \mathrm{hz}$. (Right) LiveTime as a function of the geographical coordinates.

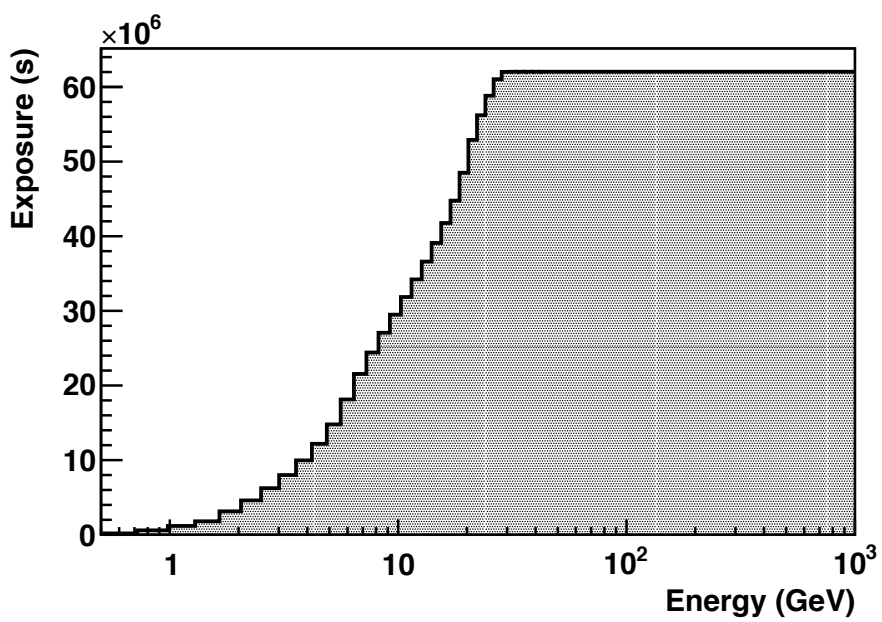

Figure 14: Exposure time corresponding to the first 30 months of data as function of the energy.

The observed drop in exposure at low energy is related to the effect of the geomagnetic field on the trajectories of incoming cosmic particles, for a given position along the orbit only particles with energy above the corresponding rigidity cutoff can reach the detector from outer space. At high energy, the time exposure is just determined by the integrated data taking time of the fully operational detector. A duty cycle of $\sim 80 \%$ is obtained in AMS, which is a remarkable results for a space experiment.

As already said in section 1.1 the measurement of electrons and positrons in the cosmic rays is very challenging due to the proton background. In order to obtained the high e/p rejection power required, the signals from TRD and ECAL are used, as explained in section 4.1, on a unitary charge sample. This unitary charge sample, is obtained through a loose preselection applied on ISS data that select only events with a relativistic down-going particle and with an associated and matching signal in TRD, Tracker and ECAL. In order to reject particles with $\mathrm{Z}>1$, the energy loss in Tracker and TRD is used as explained in section 5. Moreover the secondary events produced by the interaction of primary cosmic rays with the atmosphere, are removed from the analysis cutting over the geomagnetic cutoff. 
In the flux measurements, an electron MC sample was used in order to calculate the geometrical and the preselection acceptance. Since some details of the data, for example the interactions with the material of AMS, may not be correctly described by the MC simulation, the acceptance is corrected by a factor that take into account the difference between data and MC. In order to obtaine this factor, each cut contained in the preselection has been studied both on an electrons sample obtained from ISS data, both on electrons MC.

\section{Results}

During the first year of AMS-02 operation the main task of the collaboration has been the careful calibration of all subsystems. The objective was to keep the detector performances stable in time and at the design level in spite of the thermal excursions described in section 6 . After this first commissioning phase, the data analysis was focused on the scientific program: in the following the latest results will be briefly discussed.

\subsection{Positron fraction}

Figure 15 shows a precision measurement of the positrons fraction performed by AMS in the energy range $0.5-500 \mathrm{GeV}$.

Below $\sim 8 \mathrm{GeV}$, the positron fraction decreases with the energy and this is consistent with the standard model of the cosmic ray propagation. However, above $20 \mathrm{GeV}$, the positron fraction starts to increase with the energy, in contrast with a model that take into account only the secondary production of positrons.

In Figure 16 the comparison at high energies $(>10 \mathrm{GeV})$ of the AMS data with the previous experiment is reported: we observe that above $\sim 200 \mathrm{GeV}$ the positron fraction is no longer increasing with energy.

The analysis of the positron fraction slope behavior with energy, indicates that above $\sim 200 \mathrm{GeV}$ the positron fraction is no longer increasing with energy. The positron fraction measured by AMS02 exceeds what expected from secondary production of positrons in the ISM [21,22]. Production of positrons from nearby pulsars [23] or in dark matter annihilation [24] have been proposed to explain the experimental data, extending the positron fraction measurement to the $\mathrm{TeV}$ energy and an accurate determination of the anti-proton flux will help in disentangle the different hypotheses. These two measurements are among the main objectives of AMS.

\subsection{Electrons and positrons fluxes}

Figures 17 and 18 show respectively the electrons and positrons fluxes measured by AMS, multiplied by $\sim E^{3}$, together with the most recent measurements for comparison, as a function of the energy, up to $200 \mathrm{GeV}$ (left plot) and up to $700 \mathrm{GeV}$ (right plot).

Thank to the high statistic collected by AMS, is possible to determinate the spectral indices as a function of energy as show in Figure 19. This is a new observation and provides important information on the origins of cosmic-ray electrons and positrons. 


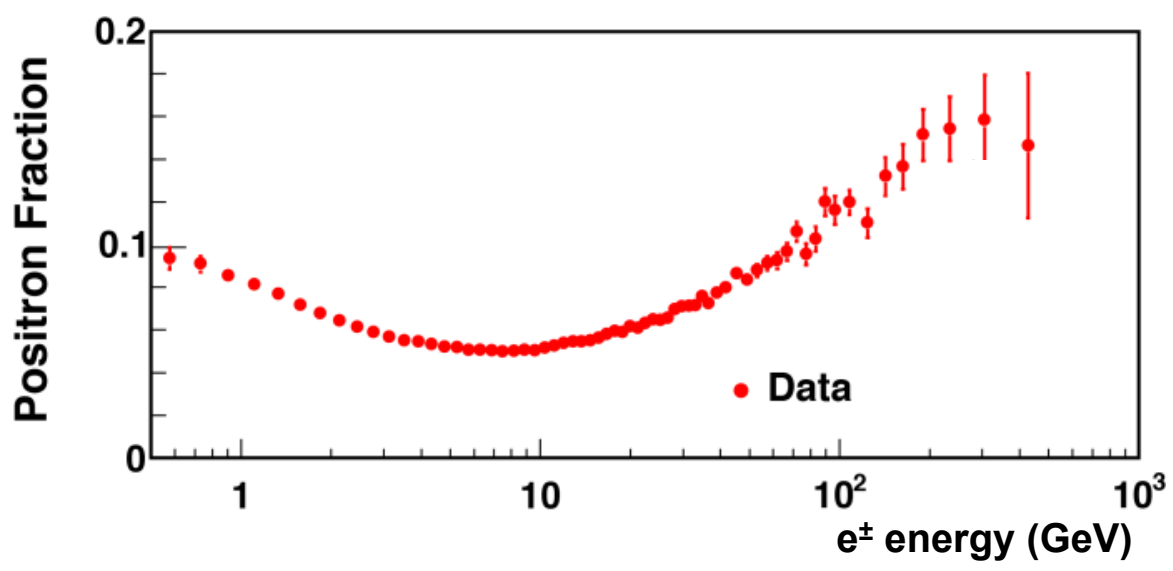

Figure 15: The positron fraction measured by AMS from 1 to $500 \mathrm{GeV}$. It shows a rapid decrease from 1 to $\sim 8 \mathrm{GeV}$ followed by a steady increase up to $\sim$ $200 \mathrm{GeV}$.

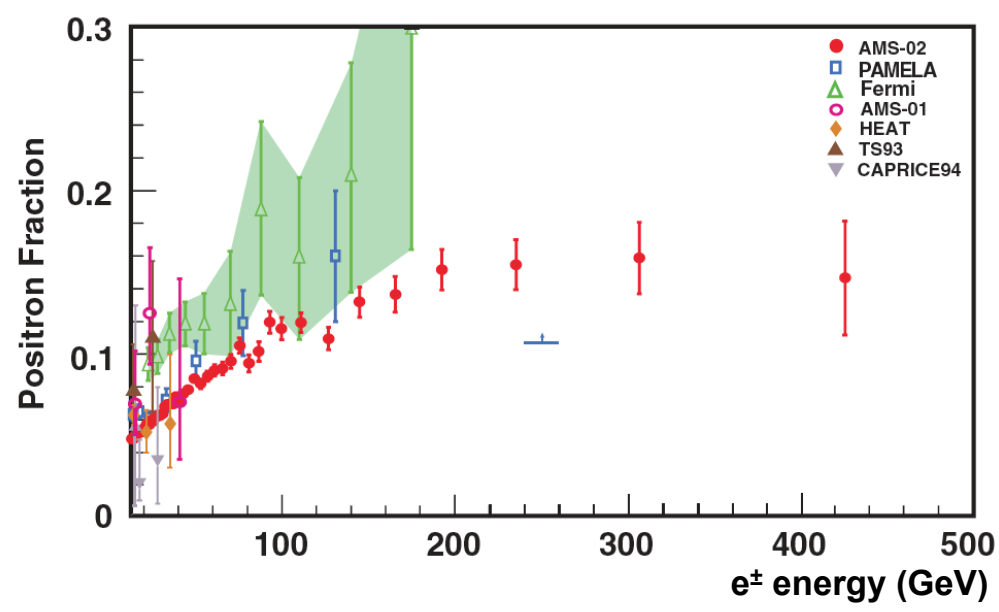

Figure 16: The positron fraction above $10 \mathrm{GeV}$, where it begins to increase. The present measurement extends the energy range to $500 \mathrm{GeV}$ and demonstrates that, above $\sim 200 \mathrm{GeV}$, the positron fraction is no longer increasing. Measurements from other experiments are also shown.

From the results, is clear that both the electrons flux and the positrons flux are significantly different in their magnitude and energy dependence and cannot be described by single power laws. The spectral indices of electrons and positrons are different, both change their behavior at $\sim 30 \mathrm{GeV}$ and show that high-energy positrons have a different origin from that of electrons. Furthermore, the rise in the positron fraction from $20 \mathrm{GeV}$ (see section 8.1) is due to an excess of positrons, not to a loss of electrons (the positron flux is harder) .

As for the positron fraction, a better understanding of the origin of the different spectral features will emerge as the flux measurements will be further extended in energy. 

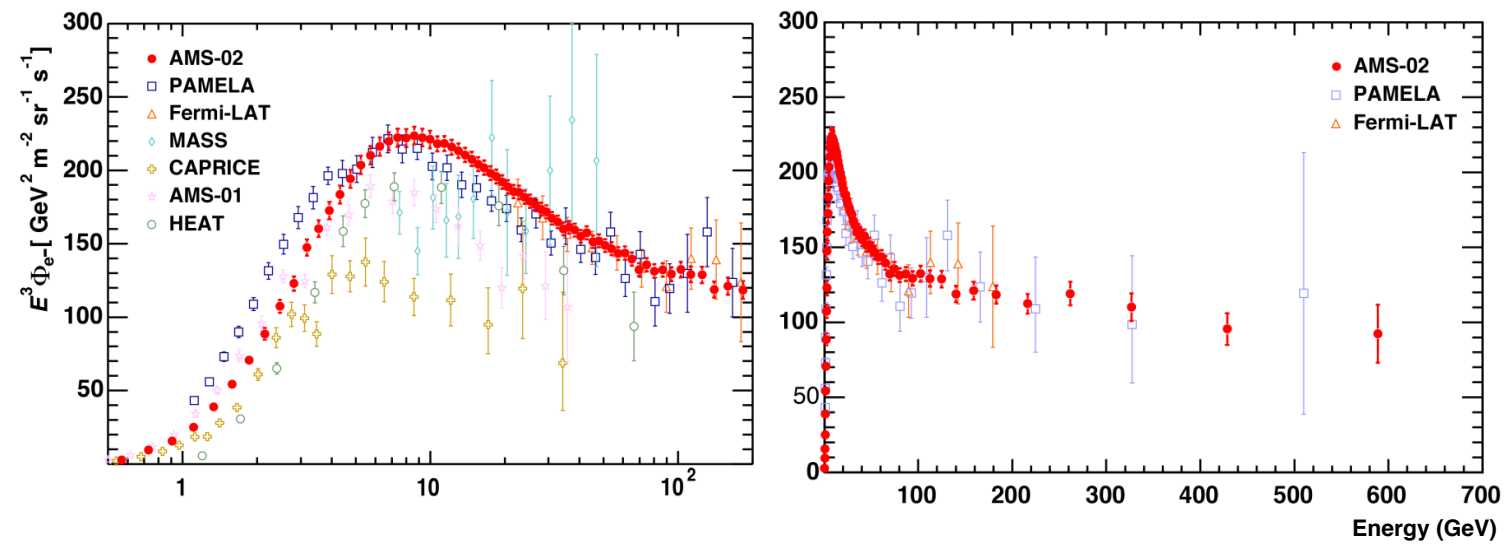

Figure 17: The AMS electrons flux, multiplied by $\mathrm{E}^{3}$ compared with the earlier measurements up to $200 \mathrm{GeV}$ (on the left) and up to $700 \mathrm{GeV}$. (on the right).
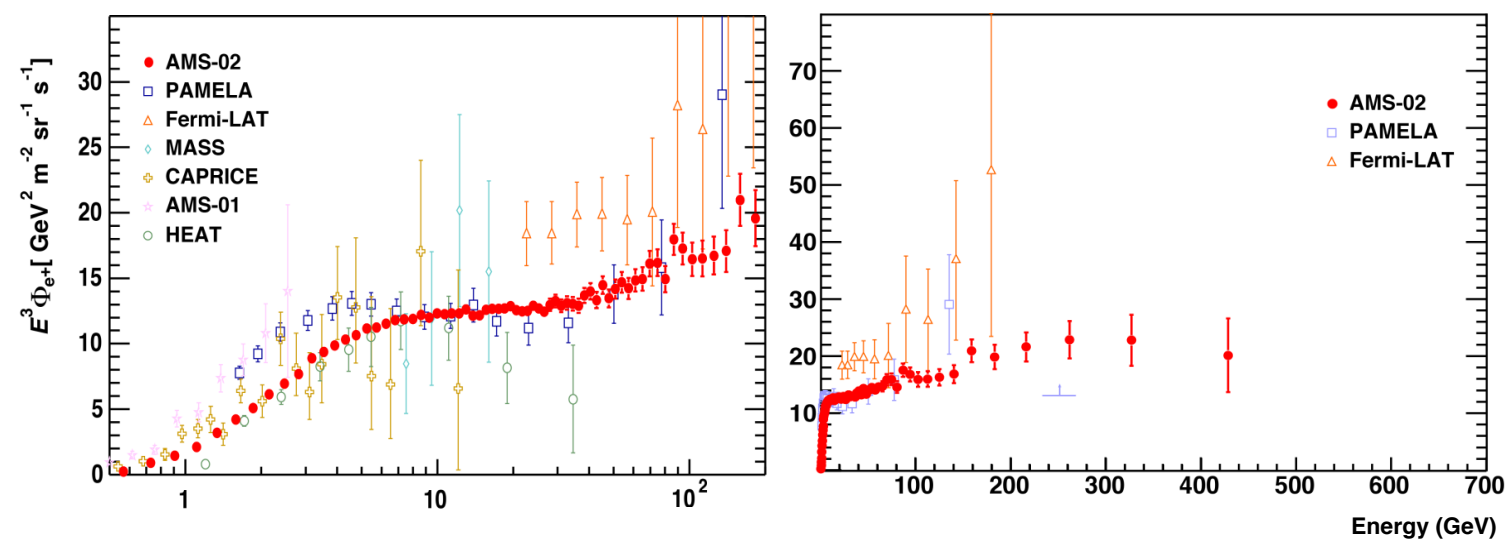

Figure 18: The AMS positrons flux, multiplied by $\mathrm{E}^{3}$ compared with the earlier measurements up to $200 \mathrm{GeV}$ (on the left) and up to $700 \mathrm{GeV}$. (on the right).

\subsection{The $\left(\mathrm{e}^{+}+\mathrm{e}^{-}\right)$flux}

The inclusive measurement of electron and positron flux has also been performed by AMS in the energy range $0.5 \mathrm{GeV}-1 \mathrm{TeV}$. In this analysis, no explicit requirement on the particle charge sign was performed; this allowed a more efficient event selection with respect to the $\mathrm{e}^{+}, \mathrm{e}^{-}$, which maximizes the statistics and minimizes any systematic uncertainty related to quality cuts in tracking and charge confusion effects. It provides a precious consistency check to the separate flux measurements presented in 8.2 and at the same time, it can be directly compared with direct measurements performed by other experiments by means of calorimetric techniques $[10,13]$ or ground based indirect measurement [cita HESS].

The measurement of $\Phi\left(e^{-}+e^{+}\right)$as a function of energy from $0.5 \mathrm{GeV}$ to $1 \mathrm{TeV}$ indicates that the flux is smooth and reveals new and distinct information. No structures were observed. From $30.2 \mathrm{GeV}$ to $1 \mathrm{TeV}$, the flux can be described by a single power law with the spectral index 


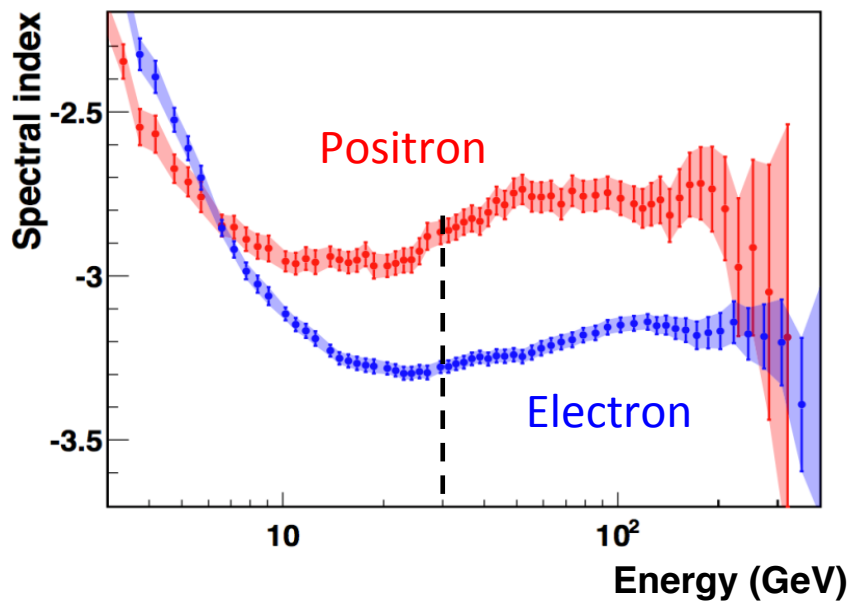

Figure 19: The spectral indices of the electron flux (blue points) and of the positron flux (red points) as a function of energy.

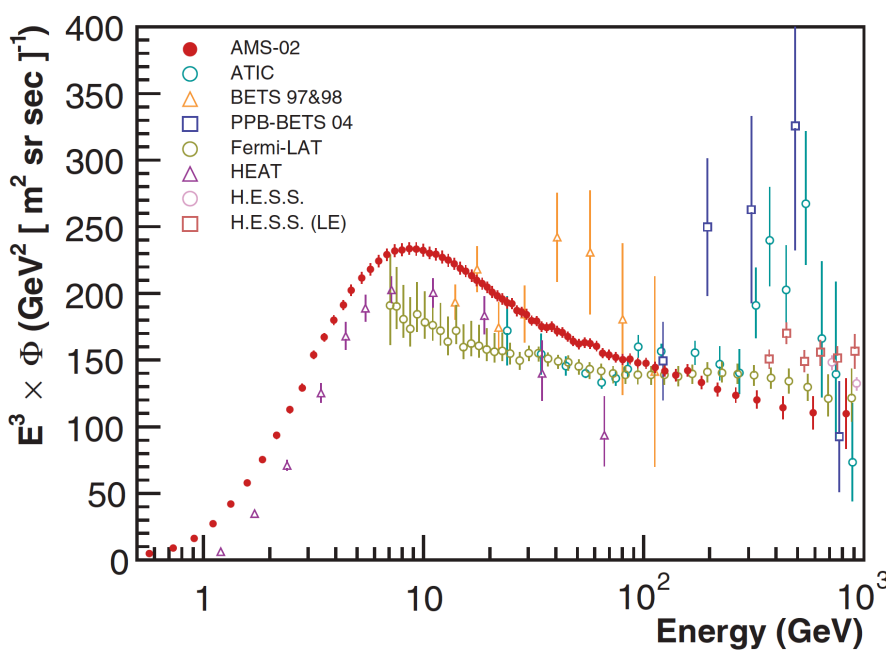

Figure 20: Electrons plus positrons flux measured by AMS multiplied by $\mathrm{E}^{3}$ as a function of energy compared with the results from earlier experiments.

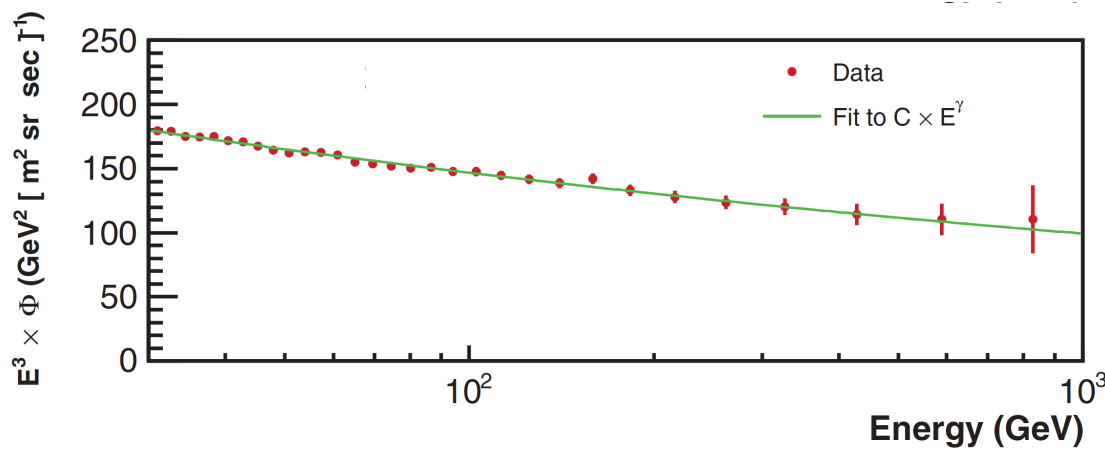

Figure 21: Fit with a single power law above $30.2 \mathrm{GeV}$ of $\Phi\left(e^{-}+e^{+}\right)$multiplied by $\mathrm{E}^{3}$.

$\gamma=-3.170 \pm 0.008$ (stat+syst) +0.008 (energy scale) as shows in Figure 21.

\section{Conclusions}

Data recorded in the first $\sim 30$ months of mission by the AMS experiment have been analyzed 
and the measurement of the energy spectra of electrons and positrons have been presented, together with the positron fraction.

The observed positron excess may imply a heavy Dark Matter WIMP particle or a new mechanism of acceleration in the pulsars. Through the measurement antiproton to proton ratio and positron fraction to high energies, AMS has the potential to shed a light on the origin of these observed features, either from exotic sources such as dark matter particles or other astrophysical sources such as pulsars.

Accurate measurements of the cosmic rays components are being performed and will allow a better tuning of the parameter that describes the Interstellar Medium and the propagation of Cosmic Rays through the galaxy.

AMS will continue its mission until ISS will be operated: it will be a Cosmic Rays observatory of the next decade.

\section{References}

[1] M. Aguilar et al., Phys. Rev. Lett. 110, 141102 (2013)

[2] M. Aguilar et al., Phys. Rev. Lett. 113, 121102 (2014)

[3] M. Aguilar et al., Phys. Rev. Lett. 113, 121102 (2014)

[4] Nucl. Instrum. Methods A 113, 221102 (2014)

[5] B. Alpat et al., Nucl. Instrum. Methods Phys. Res., Sect. A 613, 207 (2010).

[6] K. Luebelsmeyer et al., Nucl. Instrum. Methods Phys. Res., Sect. A 654, 639 (2011).

[7] Th. Kirn, Nucl. Instrum. Methods Phys. Res., Sect. A 706, 43 (2013); Ph. Doetinchem et al., Nucl. Instrum. Methods Phys.Res., Sect. A 558, 526 (2006); F. Hauler et al., IEEE Trans.Nucl. Sci. 51, 1365 (2004)

[8] B. P. Roe et al. Nucl. Instrum. Methods A 543, 2-3 (2005)

[9] O. Adriani et al. Nature 458, 607-609 (2009)

[10] M. Ackerman et al. Phys. Rev. D 82, 092004 (2010)

[11] M. Ackerman et al. Phys. Rev. Lett. 108, 011103 (2012)

[12] T.Delahaye et al., Astron. \& Astroph. 524, A51 (2010)

[13] J.Chang et al., Nature 456, 362 -365 (2008)

[14] S.Torii et al., ArXiv:0809.0760

[15] F.Aharonian et al., Phys. Rev. Lett. 101, 261104 (2008)

[16] F.Aharonian et al., A\&A 508, 561-564 (2009)

[17] O.Adriani et al., Phys. Rev. Lett. 106, 201101 (2011)

[18] Oliva, A., NIM A 588, 255 (2008)

[19] Pato, M., et al., JCAP 06, 22 (2010)

[20] J. van Es, Proc. of IAC C2.7.1 (2009) 
[21] GALPROP code. url: http://galprop.stanford.edu/.

[22] DRAGON code. url: http://dragon. hepforge.org/DRAGON/Home. html.

[23] arXiv:1304.1840 [astro-ph.HE]

[24] arXiv:1304.1184 [hep-ph] 